\title{
SEMI-BLIND RGB COLOR IMAGE WATERMARKING USING DCT AND TWO- LEVEL SVD
}

\author{
Bokan Omar Ali ${ }^{1}$ and Ghazali Bin Sulong ${ }^{2}$ \\ ${ }^{1,2}$ Department of Computer Science, Universiti Teknologi Malaysia
}

\begin{abstract}
This paper presents semi blind RGB color image watermarking using DCT and two-level SVD. First, RGB image is divided into red, green, and blue channels. The blue component is divided into blocks according to the watermark size. Second, DCT is applied to each block to form a new block in the transform domain. DC component is retrieved and assembled from each block to form a new matrix of 128x128 pixels. SVD is applied to the resultant matrix to obtain matrices, $U, S$ and $V$. The watermark is embedded into the $S$ matrix. The watermark can be extracted without original host image, however, matrices U1, S and VI are required. Experimental results indicate that the proposed algorithm can satisfy imperceptibility and it is more robust against common types of attacks such as filtering, adding noise, geometric and compression attacks.
\end{abstract}

\section{KEYWORDS}

Semi blind watermarking; Digital image watermarking; Frequency domain; Discrete Cosine Transform; singular value decomposition; attacks.

\section{INTRODUCTION}

Traditionally, developers have used analog technology to create multimedia applications. Unfortunately analog technology made it difficult to manipulate multimedia applications because of limited capacity; however, digital technologies are more flexible as well as more reliable, allowing easier handling [1].

After further progress in the field of multimedia applications and multimedia distribution, users find it difficult to protect their own content. Anyone could obtain and easily use their content as unauthorized copy. Owners need to protect their media content against theft and poor reproductive performance. Because of the wide use of the internet these types of multimedia have become unsecure. Anyone can get data from different sources and change this data without the original owner's permission. For this reason, many copyright issues have emerged recently.

Digital watermarking is a technique to embed and extract watermark information into digital data such as (images, Video or audio) to make a statement about the data. A watermark is designed to 
Signal \& Image Processing : An International Journal (SIPIJ) Vol.4, No.5, October 2013

protect multimedia data against copyright infringement or abuse [2]. Watermark information can be an image or text information about the author.

This paper is organized as follows: the problem background is discussed in Section II. In Section III the methodology is described. Experimental results are described in Section IV. The conclusion is drown in Section V.

\section{Problem BACKground}

Watermarking techniques can be classified into two parts. The first includes the spatial domain methods, which embed watermark information by directly modifying the host image pixel values. Using image's LSB to store watermark information is an example of such methods [3]. The second includes the use of transformation techniques to embed the watermark information in the frequency domain representation of the host image. The two most commonly used methods in the field of transformation are DCT and DWT [4]. DWT has better compression ratio without losing image information but it requires more processing power. DCT needs less processing power but loses a bit of image information because of blocked artefacts [5].

In a recent watermarking scheme, singular value decomposition (SVD) transformation has been used. SVD is a powerful tool used to analyze numerical matrices, resulting in a minimum least squares error truncation [6]. Image watermarking based on SVD provides secure and reliable owner identification because changing the singular values of the host image pixel does not change the host image quality much [7, 8]. SVD can embed watermark information in two ways. The first is by embedding watermark data directly into the host image pixel singular values. The second is by converting the host image into the frequency domain then embedding the watermark data in singular coefficient values [9].

SVD may be used in conjunction with DCT to improve and obtain good watermark results in terms of imperceptibility and robustness [10]. However, most previous work in this area has been non-blind, and embedded data cannot be extracted without the original host image. [10] This study proposes a non-blind watermarking schema using a combination of DCT and SVD to embed a grayscale watermark image within a grayscale host image. This method showed good performance against some attacks, however it is weak against robust cropping and median filter attacks.

Another digital image watermarking method is proposed in [13] using DWT and SVD to embed a $16 \times 16$ pixel watermark image within a RGB color host image of 200x200 pixels. This schema is robust against some types of attacks but at the same time performs poorly against Gaussian noise. A combination of DCT, SVD and edge detection image watermarking technique is used in [11]. This method is robust enough against common attacks but it is too weak against median filter and Gaussian noise. In addition, this technique depends on large blocks which decrease the capacity.

\section{PROPOSED METHOD}

In this paper, semi-blind RGB color image watermarking using a combination of DCT and two levels of SVD is proposed to generate a high degree of imperceptibility and a robust watermarked image. RGB color image of $512 \times 512$ pixels is used as a host image and a grayscale image of 
Signal \& Image Processing : An International Journal (SIPIJ) Vol.4, No.5, October 2013

$128 \times 128$ pixels is used as a watermark image. This methodology consists of three main stages including pre-processing, embedding, extracting.

First, the pre-processing stage procedure is as follows: The RGB color image is divided into three components Red (R), Green (G) and Blue (B). The Blue components have been chosen for embedding purpose as it is less sensitive to HVS. The chosen component is divided into square blocks of $(4 \times 4)$ pixels and DCT is applied to each block to form a new block in transform domain.

Second, in the embedding stage, The DC component is retrieved and assembled from each transformed block to form a new matrix of $128 \times 128$ pixels. Next, SVD is applied to the resultant matrix to get three matrices $\mathrm{U}, \mathrm{S}$ and $\mathrm{V}$ using equation (1) below:

$$
\operatorname{svd}(\mathrm{A})=\mathrm{USV}^{\mathrm{T}}
$$

Where A represents transformed matrix, $\mathrm{U}$ and $\mathrm{V}$ are two orthogonal matrices and $\mathrm{S}$ represents diagonal matrix. $\mathrm{S}$ matrix is stored for reuse in the extraction stage. Grayscale watermark image is embedded into the $\mathrm{S}$ matrix according to equation (2).

$$
\mathrm{W}^{\prime}=\mathrm{S}+\alpha \mathrm{W}
$$

Where $\mathrm{W}^{\prime}$ represents watermarked matrix, $\mathrm{S}$ is a diagonal matrix, ais scaling factor (in this paper value of $\alpha=0.1$ ) and $\mathrm{W}$ represents the watermark image. Once the process is completed, the SVD is applied to $\mathrm{W}^{\prime}$ to obtain three matrices $\mathrm{U} 1, \mathrm{~S} 1$ and $\mathrm{V} 1$. U1 and V1 and then stored for reuse in the extraction stage. Next, three matrices $\mathrm{U}, \mathrm{S} 1$ and $\mathrm{V}^{\mathrm{T}}$ are recombined to make a new matrix and each square block of $\mathrm{B}$ component is replaced with a pixel of the obtained matrix. The B channel is brought back to spatial domain using Discrete Cosine Transform (IDCT) and merged with the remaining components to form RGB color watermarked image.

Finally, the extraction stage includes extracting watermark image from the watermarked image without requiring the original host image. However, the stored matrices S, U1 and V1 are required. The procedure is as follows: RGB watermarked image is divided into three channels $\mathrm{R}$, $\mathrm{G}$ and $\mathrm{B}$. B channel is selected and divided into square blocks of $(4 \mathrm{x} 4)$ pixels and DCT is applied to each block. Extract and collect DC component from each transformed block to form a new matrix of (128x128) pixels. Perform SVD on the resultant matrix using equation (1) to create three matrices U2, S2 and V2 then obtain a new matrix by combining matrices U1, S2 and V1 transfer according to equation (3).

$$
\mathrm{D}=\mathrm{U} 1 \mathrm{~S} 2 \mathrm{~V} 1^{\mathrm{T}}
$$

Where U1 and V1 are two orthogonal matrices which were stored in the embedding stage and S2 represents a diagonal matrix. Extract watermark image pixel values by using equation (4).

$$
\mathrm{W}^{*}=\mathrm{D}-\mathrm{S} / \alpha
$$

where $\mathrm{W}^{*}$ represents extracted watermark image, D represents obtained matrix by using equation (3), S represents diagonal matrix stored during embedding stage and $\alpha$ represents scaling factor, which is $=0.1$. 
Signal \& Image Processing : An International Journal (SIPIJ) Vol.4, No.5, October 2013

Detailed algorithms for the embedding and extracting stages are as follows:

\section{A. WATERMARK EMBEDDING PROCESS}

The proposed embedding process is divided into nine steps, which are briefly described below:

Input: a dataset of standard RGB color image used as the host image is denoted by $\mathrm{H}(512 \times 512)$; a grayscale image used as a watermark image is denoted by W (128x128).

Output: RGB color watermarked image.

Step-1: Extract blue component from RGB host image H (512x512) and the selected channel is then split into square blocks of $(4 \times 4)$ pixels.

Step-2: Apply DCT to each block to obtain DCT_matrix (512x512).

Step-3: Divide DCT_matrix $(512 \times 512)$ into square blocks of $(4 \times 4)$ pixels and then extract and assemble the DC component of each block to form a new matrix A (128x128).

Step-4: Perform SVD on matrix A to obtain matrices (U, S, and V) then store matrix S to reuse during watermark extraction.

Step-5: Embed a grayscale watermark image W (128x128) pixels value in matrix $S$ according to $\mathrm{E}=\mathrm{S}+\alpha \mathrm{W}$ where $\mathrm{E}$ represents the obtained matrix, $\mathrm{S}$ represents the A singular values, $\alpha$ represents the predefined embedding strength factor. In this paper the values of $\alpha=0.1$ and $\mathrm{W}$ represent the watermark image pixels.

Step-6: Perform SVD on E to obtain (U1, S1, and V1) then store U1 and V1 to reuse in the extraction process.

Step-7: Get matrix A1 according to $\mathrm{A} 1=\mathrm{US} 1 \mathrm{~V}^{\mathrm{T}}$.

Step-8: Replace DC component of each block of DCT_matrix $(512 \times 512)$ with the A1 $(128 \times 128)$ and apply inverse DCT to each block to obtain matrix Blue_w $(512 \times 512)$.

Step-9: Recombine red, green and Blue_w components to get the RGB color watermarked image $\mathrm{H}^{*}(512 \times 512)$.

\section{B. WATERMARK EXTRACTION PROCESS}

The proposed extraction process is divided into six steps which are briefly described below:

Input: RGB color watermarked image; S, U1 and V1 matrices.

Output: Extracted grayscale watermark image.

Step-1: Extract blue component from RGB watermarked image $H^{*}(512 \times 512)$ and then divide the selected channel into square blocks of $(4 \mathrm{x} 4)$ pixels. 
Step-2: Apply DCT on each block to obtain DCT_matrix (512x512).

Step-3: Divide DCT_matrix $(512 \times 512)$ into square blocks of $(4 \times 4)$ pixels and then extract and collect DC component from each block to form a new matrix B (128x128).

Step-4: Perform SVD on the matrix B to obtain matrices (U2, S2, and V2).

Step-5: Obtain matrix D according to $\mathrm{D}=\mathrm{U} 1 \mathrm{~S} 2 \mathrm{~V} 1^{\mathrm{T}}$.

Step-6: Finally, extract watermark pixel values according to $\mathrm{W}^{*}=\mathrm{D}-\mathrm{S} / \alpha$.

\section{EXPERIMENTAL RESULTS AND DISCUSSIONS}

In this study, a dataset of standard RGB color image of $(512 \times 512)$ pixels is used as a host image and a grayscale image of $128 \times 128$ pixels is used as a watermark image. Peak signal to Noise Ratio (PSNR) is used to measure the quality ratio between cover the image and its watermark. Normalized cross correlation (NCC) is used to define the similarity between the original and extracted watermarks. Due to limited space, here we just show the results obtained using two selected dataset images which are (Lena.jpg and Pepper.jpg). Different attacks such as filtering (median filter, sharpen filter, motion blur), adding noise (salt \& pepper noise, Gaussian, Poisson, speckle), geometric attacks (rotation and cropping) and compression attacks (JPEG compression) are used to test the proposed algorithm in term of robustness. Figure 1 shows the original watermark image. Figures 2 and 3 the selected dataset of standard RGB color host images and watermarked images with their respective PSNR value. The extracted watermark images under various types of attacks with their NCC values are shown in Figures 4, 5 and 6. The comparison between the proposed method and existing methods in [12] and [13] is shown in Table 1.

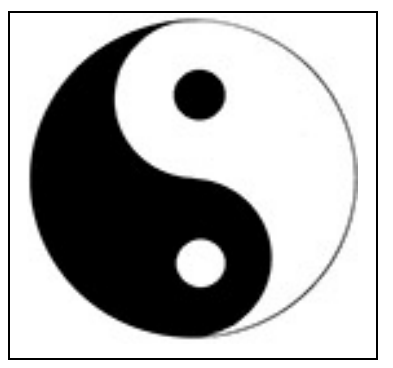

Fig 1. Original watermark image 
Signal \& Image Processing : An International Journal (SIPIJ) Vol.4, No.5, October 2013

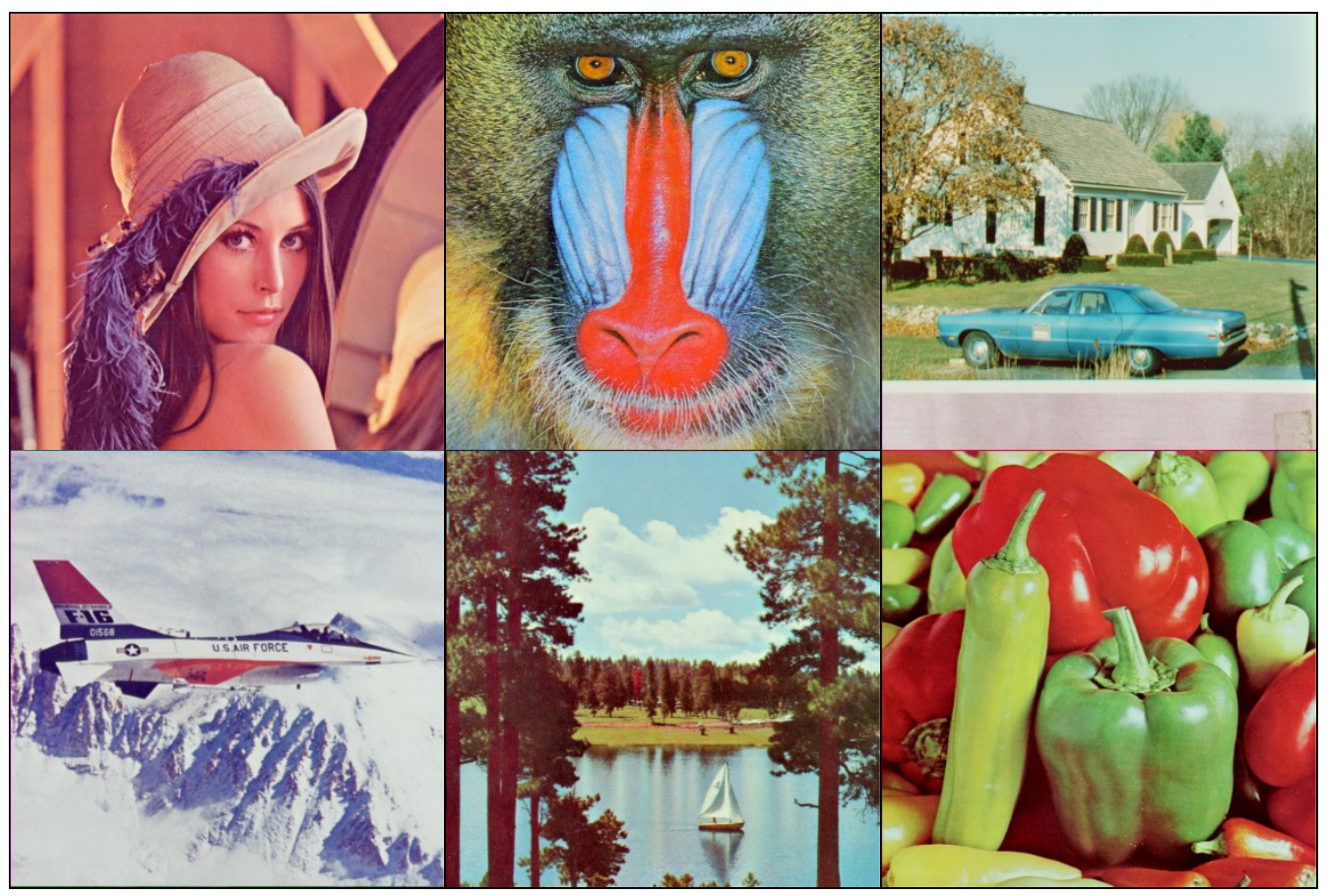

Fig 2. Standard dataset of RGB host image

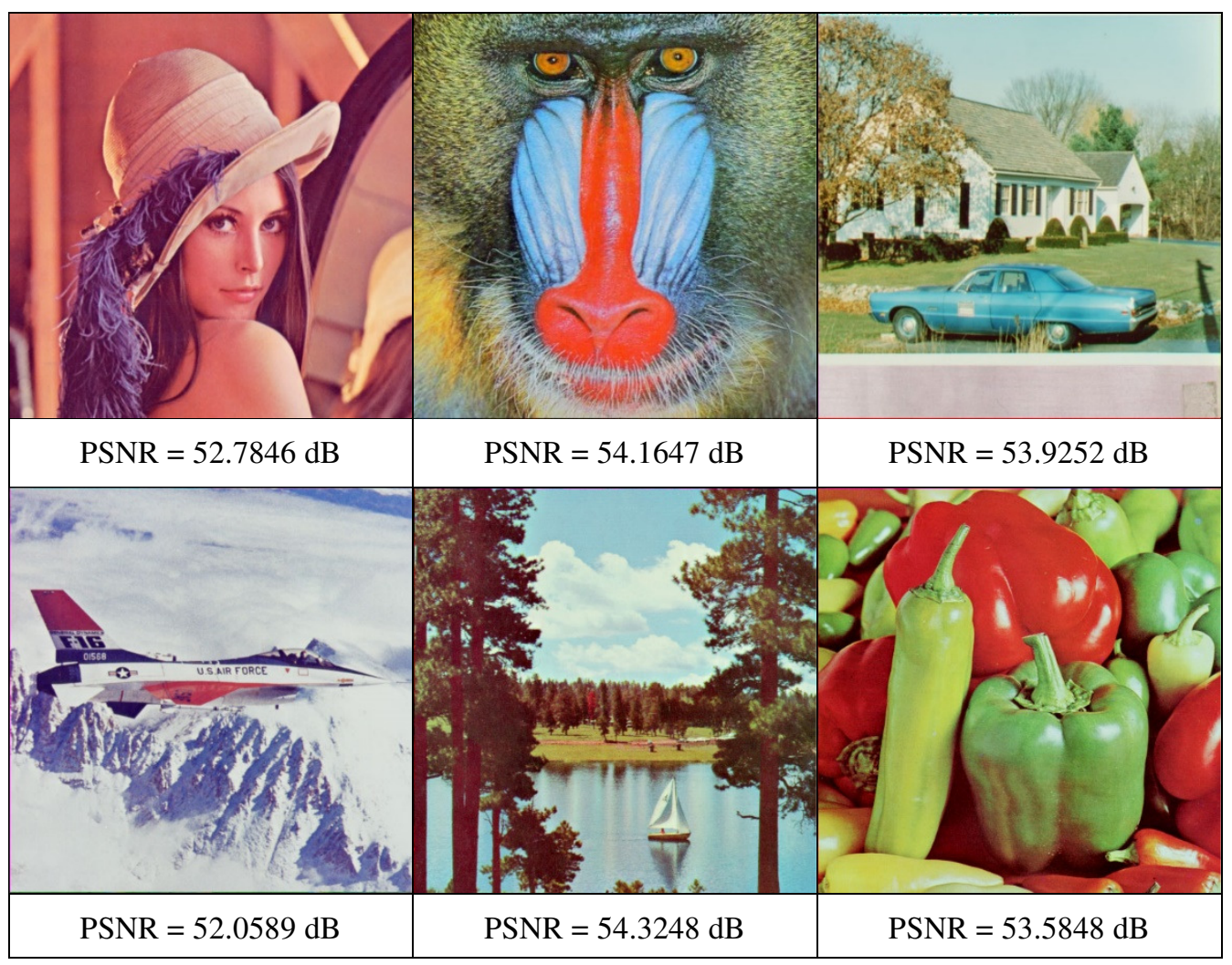

Fig 3. RGB watermarked images with their PSNR values 
Signal \& Image Processing : An International Journal (SIPIJ) Vol.4, No.5, October 2013

PSNR values for the watermarked images are very high which means that the cover image and its watermarked are almost identical.

\begin{tabular}{|c|c|c|c|c|}
\hline Attacks & $\begin{array}{c}\text { Extracted watermark } \\
\text { image from Lena.jpg } \\
\text { host image }\end{array}$ & NCC & $\begin{array}{c}\text { Extracted watermark } \\
\text { image from Pepper.jpg } \\
\text { host image }\end{array}$ & NCC \\
\hline Sharpen filter & 0.9902 & & 0.9900 \\
\hline Median filter 3x3 & & 0.9855 & & 0.9849 \\
\hline Motion Blur & & 0.8317 & & \\
\hline
\end{tabular}

Fig 4. Extracted watermark image with their NCC value after attacking watermarked host image with various types of filter attack.

\begin{tabular}{|c|c|c|c|c|}
\hline Attacks & $\begin{array}{c}\text { Extracted watermark } \\
\text { image from Lena.jpg } \\
\text { host image }\end{array}$ & NCC & $\begin{array}{c}\text { Extracted watermark } \\
\text { image from Pepper.jpg } \\
\text { host image }\end{array}$ & NCC \\
\hline $\begin{array}{c}\text { Salt \& Pepper } \\
\text { noise } 0.01\end{array}$ & 0.9889 & & 0.9830 \\
\hline $\begin{array}{c}\text { Gaussian noise } \\
0.01\end{array}$ & 0.9884 & & & 0.9614 \\
\hline $\begin{array}{c}\text { Speckle noise } \\
0.01\end{array}$ & 0.9945 & & & \\
\hline
\end{tabular}

Fig 5. Extracted watermark image with their NCC value after attacking watermarked host image with various types of adding noise attack. 
Signal \& Image Processing : An International Journal (SIPIJ) Vol.4, No.5, October 2013

\begin{tabular}{|c|c|c|c|c|}
\hline Attacks & $\begin{array}{c}\text { Extracted watermark } \\
\text { image from Lena.jpg } \\
\text { host image }\end{array}$ & NCC & $\begin{array}{c}\text { Extracted watermark } \\
\text { image from Pepper.jpg } \\
\text { host image }\end{array}$ & NCC \\
\hline Rotate $90^{\circ}$ & & 0.9995 & & 0.9971 \\
\hline Cropping $25 \%$ & & 0.9681 & & 0.8460 \\
\hline JPEG (50) quality & & 0.9916 & & 0.9819 \\
\hline JPEG (80) quality & & 0.9941 & & 0.9808 \\
\hline
\end{tabular}

Fig 6. Extracted watermark image with their NCC value after attacking watermarked host image with various types of geometric attack.

Normalized cross correlation is used to analyze the differences between the original watermark images and the recovered watermark images which have passed various attacks such as filtering attacks, adding noise attacks and geometric attacks. NCC results show that the proposed method has excellent resistance to most common attacks such as sharpening, median filter, salt and pepper, Poisson noise, cropping, rotation, and JPEG compression.

Table 1: Robustness performance: proposed method verses methods in [12] and [13].

\begin{tabular}{|c|c|c|c|}
\hline Methods & $\begin{array}{c}\text { Proposed } \\
\text { method }\end{array}$ & Reference[12] & Reference[13] \\
\hline Sharpen filter & 0.9902 & 0.6128 & $\mathrm{n} / \mathrm{a}$ \\
\hline Median filter 3x3 & 0.9855 & 0.6842 & 0.8968 \\
\hline Motion Blur & 0.8317 & $\mathrm{n} / \mathrm{a}$ & $\mathrm{n} / \mathrm{a}$ \\
\hline Salt \& Pepper noise 0.01 & 0.9889 & 0.9492 & $\mathrm{n} / \mathrm{a}$ \\
\hline Gaussian noise 0.01 & 0.9884 & 0.8365 & 0.7415 \\
\hline Speckle noise 0.01 & 0.9945 & $\mathrm{n} / \mathrm{a}$ & $\mathrm{n} / \mathrm{a}$ \\
\hline Poisson noise & 0.9954 & $\mathrm{n} / \mathrm{a}$ & $\mathrm{n} / \mathrm{a}$ \\
\hline Rotation 90 & 0.9995 & $\mathrm{n} / \mathrm{a}$ & $\mathrm{n} / \mathrm{a}$ \\
\hline Crop 25\% & 0.9681 & 0.8473 & 0.9031 \\
\hline JPEG 50 & 0.9916 & 0.8150 & 0.9402 \\
\hline JPEG 80 & 0.9941 & 0.8610 & $\mathrm{n} / \mathrm{a}$ \\
\hline
\end{tabular}


Signal \& Image Processing : An International Journal (SIPIJ) Vol.4, No.5, October 2013

Based on the comparison of the proposed method and the existing works in [12] and [13], the proposed method is more resistant to the most common attacks when compared to the previous works.

\section{CONCLUSION}

This paper offered semi blind color image watermarking based on DCT and two-level SVD. The proposed method has an excellent resistance to common types of attacks such as filtering, adding noise and geometric attacks. Without referring to the original host image an embedded watermark can be extracted from an attacked watermarked image. The experimental results have shown that the proposed algorithm has a strong resistance to common image processing operations. A comparison of the proposed method and the existing methods in [12] and [13] shows that the proposed method has better performance.

\section{REFERENCES}

[1] Friedman, G. L. (1993). The trustworthy digital camera: Restoring credibility to the photographic image. Consumer Electronics, IEEE Transactions on, 39(4), 905-910.

[2] Dharwadkar, N. V., and Amberker, B. (2010). Watermarking Scheme for Color Images using Wavelet Transform based Texture Properties and Secret Sharing. International Journal of Signal Processing, 6(2).

[3] Chang, C.-C., Hsiao, J.-Y., and Chan, C.-S. (2003). Finding optimal least-significant-bit substitution in image hiding by dynamic programming strategy. Pattern Recognition, 36(7), 1583-1595.

[4] Barni, M., Bartolini, F., Cappellini, V., and Piva, A. (1998). A DCT-domain system for robust image watermarking. Signal processing, 66(3), 357-372.

[5] Nadenau, M. (2000). Integration of human color vision models into high quality image compression. École Polytechnique Fédérale De Lausanne, Thesis No, 2296.

[6] Liu, F., and Qian, Y. (2011). A Novel Robust Watermarking Algorithm Based On Two_Levels DCT and Two_Levels SVD. Paper presented at the Measuring Technology and Mechatronics Automation (ICMTMA), 2011 Third International Conference on.

[7] Liu, F., Han, K., and zheng Wang, C. (2009). A novel blind watermark algorithm based On SVD and DCT. Paper presented at the Intelligent Computing and Intelligent Systems, 2009. ICIS 2009. IEEE International Conference on.

[8] Shi, F., Shi, Y., and Lai, L. (2011). Optimization on digital watermarking algorithm based on SVDDWT. Paper presented at the Granular Computing (GrC), 2011 IEEE International Conference on.

[9] Li, Z., Yap, K.-H., and Lei, B.-Y. (2011). A new blind robust image watermarking scheme in SVDDCT composite domain. Paper presented at the Image Processing (ICIP), 2011 18th IEEE International Conference on.

[10] Quan, L., and Qingsong, A. (2004). A combination of DCT-based and SVD-based watermarking scheme. Paper presented at the Signal Processing, 2004. Proceedings. ICSP'04. 2004 7th International Conference on.

[11] Rajani, A., and Ramashri, T. (2011). Image Watermarking Algorithm Using DCT, SVD and Edge Detection Technique. International Journal of Engineering Research and Applications (IJERA), l(4), 1828-1834.

[12] Golea, N. H., Seghir, R. and Benzid, R. (2010). A blind RGB color image watermarking based on singular value decomposition. In Computer System and Applications (AICCSA), IEEE/ACS International Conference on.

[13] Yin, C. Q., Li, L., Lv, A. Q. and Qu, L. (2007, August). Color image watermarking algorithm based on DWT-SVD. In Automation and Logistics, 2007 IEEE International Conference on (pp. 26072611). 
Signal \& Image Processing : An International Journal (SIPIJ) Vol.4, No.5, October 2013

\section{AUTHORS}

Bokan Omar Ali was born in Jan 1, 1985, Kalar, Kurdistan-Iraq. He received his B.Sc. Computer Science 2006 University of Duhok, Kurdistan-Iraq. Currently he is M.Sc. student in University Technology Malaysia (UTM), Johor, Malaysia.

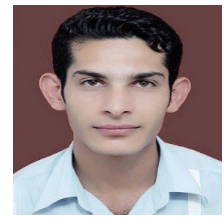

Ghazali Bin Sulong was born in May 21, 1958 Malaysia. He received his Ph.D. Computing 1989 University of Wales College of Cardiff (UWCC), Wales, U.K., M.Sc. Computing 1982 University of Wales College Cardiff (UCC), Wales, U.K., B.Sc. Statistic 1979 UKM, Malaysia. Currently he is a Professor in Image Processing.

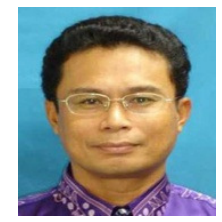

